\title{
An Area Preserving Projection from the Regular Octahedron to the Sphere
}

\author{
Daniela Roşca and Gerlind Plonka
}

\author{
Dedicated to Werner Haußmann in memoriam
}

\begin{abstract}
In this paper, we propose an area preserving bijective map from the regular octahedron to the unit sphere $\mathbb{S}^{2}$, both centered at the origin. The construction scheme consists of two steps. First, each face $F_{i}$ of the octahedron is mapped to a curved planar triangle $\mathcal{T}_{i}$ of the same area. Afterwards, each $\mathcal{T}_{i}$ is mapped onto the sphere using the inverse Lambert azimuthal equal area projection with respect to a certain point of $\mathbb{S}^{2}$. The proposed map is then used to construct uniform and refinable grids on a sphere, starting from any triangular uniform and refinable grid on the triangular faces of the octahedron.
\end{abstract}

Mathematics Subject Classification (2000). Primary 86A30; Secondary 85-08, 86-08.

Keywords. Equal area projection, uniform spherical grid, refinable grid, hierarchical grid.

\section{Introduction}

In many applications, especially in geosciences and astronomy, but also in computer vision, one is interested in simple, refinable grids on the sphere. In particular, one requires partitions of $\mathbb{S}^{2}$ into regions of equal area and small diameter in order to avoid the distortions that often occur in statistical computations and function approximations using non-equal area partitions.

There exist already some constructions of equal area partitions of $\mathbb{S}^{2}$. Based on the construction by Zhou [12], Leopardi [4] derives a recursive zonal equal area partition of the unit sphere $\mathbb{S}^{d} \subset \mathbb{R}^{d+1}$ that consists of polar cups

This work has been supported by the German Research Foundation, Grant PL 170/14-1. 
and rectilinear regions. This construction has the disadvantage that one has to deal with different kinds of areas. The existence of partitions of $\mathbb{S}^{2}$ into regions of equal area and small diameter has been already used by Alexander [1], who derived lower bounds for the maximum sum of distances between points on the sphere. Tegmark [11] considered an icosahedron-based pixelizing of the sphere. Similarly, the algorithm for binning globally distributed measurements on the sphere by Teanby [10] is based on repeated subdivision of the icosahedron. In [8], a subdivision method is proposed using a spherical triangulation that is obtained by projecting the faces of an icosahedron to the sphere. The HEALPix (hierarchical equal area iso-latitude pixelization) introduced in [3], has been frequently used in recent years for application of local pixel set operations, multiresolution applications and fast spherical harmonic transforms.

However, one simple method to construct grids on the sphere is to transfer existing planar grids by a suitable projection. For a survey of known spherical projections from the sphere or parts of the sphere to the plane we refer to $[2,9]$. Therefore we are especially interested in the construction of equal area partitions being obtained by application of an area-preserving bijective map from suitable planar domains to (parts of) the sphere.

In [5], one of the authors already suggested a new area preserving projection method based on a mapping of the square onto a disc in a first step, followed by a lifting to the sphere by the inverse Lambert projection. This idea can also be generalized to construct uniform and refinable grids on elliptic domains and on some surfaces of revolution, see [6]. In [7], the authors have recently constructed an equal area projection from the cube to the sphere.

In this paper we construct an area preserving map from a regular octahedron to the unit sphere $\mathbb{S}^{2}$. Thus, any grid on the octahedron can be transported to the sphere. Further, since arbitrary grids on the triangle can now simply be transported to the sphere, we believe that this construction may achieve an essential impact for different applications in geosciences. Since we give explicit formulas both for the map from the octahedron to the sphere, and from the sphere to the octahedron, the method is easy to implement.

The construction scheme consists of two steps. In the first step, we construct in Sect. 3 an area preserving bijection $U$ from each face $F_{i}$ of the octahedron, onto a planar domain $\widetilde{F}_{i}$, bounded by a curved triangle $\mathcal{T}_{i}$. In the second step, we combine $U$ with an inverse Lambert azimuthal projection, in order to map each face $F_{i}$ of the octahedron onto a subset $\mathcal{F}_{i}$ of the sphere, such that $\cup_{i=1}^{8} \overline{\mathcal{F}_{i}}=\mathbb{S}^{2}$. A closed form of the inverse area preserving map $U^{-1}$ is presented in Sect. 6. Further, we present some examples of the obtained spherical grids.

\section{Preliminaries}

Consider the unit sphere $\mathbb{S}^{2}$ centered at the origin $O$ and the regular octahedron $\mathbb{K}$ of the same area, centered at $O$ and with vertices on the coordinate 


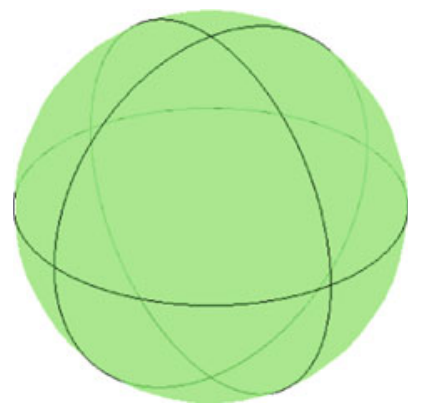

FiguRE 1. The eight spherical triangles obtained as intersection of the coordinate planes with the sphere

axes. Since the area of the sphere is $4 \pi$, the area of each face of $\mathbb{K}$ is $\pi / 2$ and the edge of the octahedron has the length

$$
\ell=\frac{\sqrt{2 \pi}}{\sqrt[4]{3}} .
$$

We cut the sphere with the coordinate planes $x=0, y=0, z=0$ and obtain the spherical triangles in Fig. 1. Each face $F_{i}, i=1, \ldots, 8$, of the octahedron $\mathbb{K}$ is thus situated in one of the following domains

$$
\begin{array}{ll}
I_{1}=\{(x, y, z), x \geq 0, y \geq 0, z \geq 0\}, \quad I_{2}=\{(x, y, z), x \geq 0, y \geq 0, z \leq 0\}, \\
I_{3}=\{(x, y, z), x \geq 0, y \leq 0, z \geq 0\}, \quad I_{4}=\{(x, y, z), x \geq 0, y \leq 0, z \leq 0\}, \\
I_{5}=\{(x, y, z), x \leq 0, y \geq 0, z \geq 0\}, \quad I_{6}=\{(x, y, z), x \leq 0, y \geq 0, z \leq 0\}, \\
I_{7}=\{(x, y, z), x \leq 0, y \leq 0, z \geq 0\}, \quad I_{8}=\{(x, y, z), x \leq 0, y \leq 0, z \leq 0\} .
\end{array}
$$

More precisely, $F_{i} \subset I_{i}$ for $i=1, \ldots, 8$ and the portion of the sphere situated in $I_{i}$ will be denoted by $\mathcal{F}_{i}$, i.e.,

$$
\mathcal{F}_{i}=\left\{(x, y, z) \in I_{i}, x^{2}+y^{2}+z^{2}=1\right\}
$$

see Fig. 1.

We focus on the portion $\mathcal{F}_{1}$ of the sphere and consider its center point $G_{1}=(\nu, \nu, \nu)$, with $\nu=1 / \sqrt{3}$. The point $G_{1}$ is in fact the intersection of the line $O G_{1}^{\prime}$ with $\mathbb{S}^{2}$, where $G_{1}^{\prime}$ is the center of mass of the face $F_{1}$.

We consider the Lambert projection $L_{G_{1}}$ of the domain $\mathcal{F}_{1} \subset \mathbb{S}^{2}$ with respect to the point $G_{1}$. Thus, $\mathcal{F}_{1}$ will be mapped on a curved triangle that is situated in the plane $x+y+z=\sqrt{3}$ perpendicular to the line $O G_{1}$. For calculating this projection, we apply a transformation to the sphere such that $G_{1}$ is mapped onto the South Pole $S=(0,0,-1)$. Such a transformation $\mathcal{R}$ consists in two successive rotations, $\mathcal{R}_{1}$ and $\mathcal{R}_{2}$. The first rotation $\mathcal{R}_{1}$ is taken 
around the $z$-axis with angle $-\pi / 4$ and maps $G_{1}$ onto $P_{1}=\left(\frac{\sqrt{2}}{\sqrt{3}}, 0, \frac{1}{\sqrt{3}}\right)$. The second rotation $\mathcal{R}_{2}$ is around the $y$-axis and maps $P_{1}$ onto $S=(0,0,-1)$, so its angle is $\beta+\frac{\pi}{2}$, with $\beta=\arcsin \frac{1}{\sqrt{3}}$. The corresponding rotation matrices of $\mathcal{R}_{1}, \mathcal{R}_{2}$ and $\mathcal{R}$ have the form

$$
\begin{aligned}
& \mathcal{R}_{1}=\left(\begin{array}{ccc}
\cos \frac{\pi}{4} & \sin \frac{\pi}{4} & 0 \\
-\sin \frac{\pi}{4} & \cos \frac{\pi}{4} & 0 \\
0 & 0 & 1
\end{array}\right)=\left(\begin{array}{ccc}
\frac{1}{\sqrt{2}} & \frac{1}{\sqrt{2}} & 0 \\
-\frac{1}{\sqrt{2}} & \frac{1}{\sqrt{2}} & 0 \\
0 & 0 & 1
\end{array}\right), \\
& \mathcal{R}_{2}=\left(\begin{array}{ccc}
\cos \left(\beta+\frac{\pi}{2}\right) & 0 & \sin \left(\beta+\frac{\pi}{2}\right) \\
0 & 1 & 0 \\
-\sin \left(\beta+\frac{\pi}{2}\right) & 0 \cos \left(\beta+\frac{\pi}{2}\right)
\end{array}\right)=\left(\begin{array}{ccc}
-\frac{1}{\sqrt{3}} & 0 & \frac{\sqrt{2}}{\sqrt{3}} \\
0 & 1 & 0 \\
-\frac{\sqrt{2}}{\sqrt{3}} & 0 & -\frac{1}{\sqrt{3}}
\end{array}\right), \\
& \mathcal{R}=\mathcal{R}_{2} \cdot \mathcal{R}_{1}=\left(\begin{array}{ccc}
-\frac{1}{\sqrt{6}} & -\frac{1}{\sqrt{6}} & \frac{\sqrt{2}}{\sqrt{3}} \\
-\frac{1}{\sqrt{2}} & \frac{1}{\sqrt{2}} & 0 \\
-\frac{1}{\sqrt{3}} & -\frac{1}{\sqrt{3}} & -\frac{1}{\sqrt{3}}
\end{array}\right) .
\end{aligned}
$$

The matrices $\mathcal{R}_{1}, \mathcal{R}_{2}$ and $\mathcal{R}$ are orthogonal, therefore $\mathcal{R}^{-1}=\mathcal{R}^{T}$.

Now, in order to obtain the Lambert projection $L_{G_{1}}$ with respect to $G_{1} \in \mathbb{S}^{2}$, we use the azimuthal Lambert projection $L_{S}$ with respect to the South Pole $S$, as follows:

$$
L_{G_{1}}=\mathcal{R}^{-1} \circ L_{S} \circ \mathcal{R} .
$$

It is well known that the image of a point $(x, y, z) \in \mathbb{S}^{2}$ by $L_{S}$ onto the tangential plane at the South Pole is

$$
\left(\sqrt{\frac{2}{1-z}} x, \sqrt{\frac{2}{1-z}} y,-1\right)
$$

For the rotated Lambert projection on $Z_{L}=-1$ we obtain

$$
\left(L_{S} \circ \mathcal{R}\right)(x, y, z)=\left(X_{L}, Y_{L},-1\right)=\left(\frac{\nu(2 z-x-y)}{\sqrt{1+\nu(x+y+z)}}, \frac{\nu(-x+y)}{\sqrt{1+\nu(x+y+z)}},-1\right)
$$

with $\nu=1 / \sqrt{3}$. Therefore, for $L_{G_{1}}$ we find

$$
L_{G_{1}}(x, y, z)=\left(\nu+\frac{\sqrt{2} \nu^{2}(2 x-y-z)}{\sqrt{1+\nu(x+y+z)}}, \nu+\frac{\sqrt{2} \nu^{2}(2 y-x-z)}{\sqrt{1+\nu(x+y+z)}}, \nu+\frac{\sqrt{2} \nu^{2}(2 z-x-y)}{\sqrt{1+\nu(x+y+z)}}\right) .
$$

Let us denote by $l_{1}, l_{2}, l_{3}$ the edges of the spherical triangle $\mathcal{F}_{1}$, situated on the planes $z=0, y=0$ and $x=0$, respectively, see Fig. 1 . These edges are mapped by $L_{S} \circ \mathcal{R}$ onto the planar curves in the plane $Z_{L}=-1$, given by the following parametric equations 


$$
\begin{aligned}
& \left(L_{S} \circ \mathcal{R}\right)\left(l_{1}\right):\left\{\begin{array}{l}
X_{L}=\frac{(-x-y)}{\sqrt{3+\sqrt{3}(x+y)}}, \text { with } y=\sqrt{1-x^{2}}, x \in[0,1], \\
Y_{L}=\frac{\sqrt{3}(-x+y)}{\sqrt{3+\sqrt{3}(x+y)},},
\end{array}\right. \\
& \left(L_{S} \circ \mathcal{R}\right)\left(l_{2}\right):\left\{\begin{array}{l}
X_{L}=\frac{(2 z-x)}{\sqrt{3+\sqrt{3}(x+z)}}, \text { with } z=\sqrt{1-x^{2}}, x \in[0,1], \\
Y_{L}=\frac{-\sqrt{3} x}{\sqrt{3+\sqrt{3}(x+z)}},
\end{array}\right. \\
& \left(L_{S} \circ \mathcal{R}\right)\left(l_{3}\right):\left\{\begin{array}{l}
X_{L}=\frac{(2 z-y)}{\sqrt{3+\sqrt{3}(y+z)}} \text { with } z=\sqrt{1-y^{2}}, y \in[0,1] . \\
Y_{L}=\frac{\sqrt{3} y}{\sqrt{3+\sqrt{3}(y+z)}},
\end{array}\right.
\end{aligned}
$$

We denote the curved triangle determined by these three curves by $\mathcal{T}_{1}=$ $\mathcal{T}$. It can be simply transferred to the plane $x+y+z=\sqrt{3}$ using the rotation map $\mathcal{R}^{-1}$ (and hence finishing the projection $L_{G_{1}}$ ). But we prefer to compare the curved triangle $\mathcal{T}$ with the rotated triangular face $T=\mathcal{R} T_{1}$ of the octahedron in the plane $Z_{L}=-1$. A simple calculation shows that the vertices of the equilateral triangle $T=\mathcal{R} T_{1}$ are $P_{1}=(2 \alpha, 0,-1), P_{2}=(-\alpha, \ell / 2,-1)$, and $P_{3}=(-\alpha,-\ell / 2,-1)$, with

$$
\alpha=\sqrt{\frac{\pi}{6 \sqrt{3}}}
$$

and side length $\ell$ in (2.1). Figure 2 (left) shows the curved triangle $\mathcal{T}$ and the equilateral triangle $T$ of the same area $\pi / 2$. With the help of the projection $L_{G_{1}}$ (resp. $\left.L_{S} \circ \mathcal{R}\right)$, we have simplified now the problem of finding an area preserving map from the octahedron to the sphere $\mathbb{S}^{2}$ to a problem of finding a two-dimensional map from an equilateral triangle $T$, i.e., a face of the octahedron, to the curved triangle $\mathcal{T}$. In our further considerations in Sect. 3, we will consider only two dimensions while $Z_{L}=-1$ is fixed.

\section{Mapping a Triangle onto a Curved Triangle}

In this section we derive an area preserving bijection $U: \mathbb{R}^{2} \rightarrow \mathbb{R}^{2}$, which maps the equilateral triangle $T$ with vertices $P_{1}=(2 \alpha, 0), P_{2}=(-\alpha, \ell / 2)$, and $P_{3}=(-\alpha,-\ell / 2)$ onto the curved triangle $\mathcal{T}$ that has been constructed in Sect. 2. Here, we say that the map $U$ is area-preserving, if it has the property

$$
\mathcal{A}(D)=\mathcal{A}(U(D)), \text { for every domain } D \subseteq \mathbb{R}^{2},
$$

where $\mathcal{A}(D)$ denotes the area of $D$.

We consider the three line segments $h_{1}=O P_{1}, h_{2}=O P_{2}, h_{3}=O P_{3}$, that form the angles $0,2 \pi / 3,4 \pi / 3$ around $O$, see Fig. 2(left), and determine three disjoint regions of $\mathbb{R}^{2}$ defined by

$$
\begin{aligned}
& \mathcal{Q}_{1}=\left\{(x, y) \in \mathbb{R}^{2}, \quad \sqrt{3} x \leq y \leq-\sqrt{3} x\right\}, \\
& \mathcal{Q}_{2}=\left\{(x, y) \in \mathbb{R}^{2}, \quad y<0, y \leq \sqrt{3} x\right\},
\end{aligned}
$$



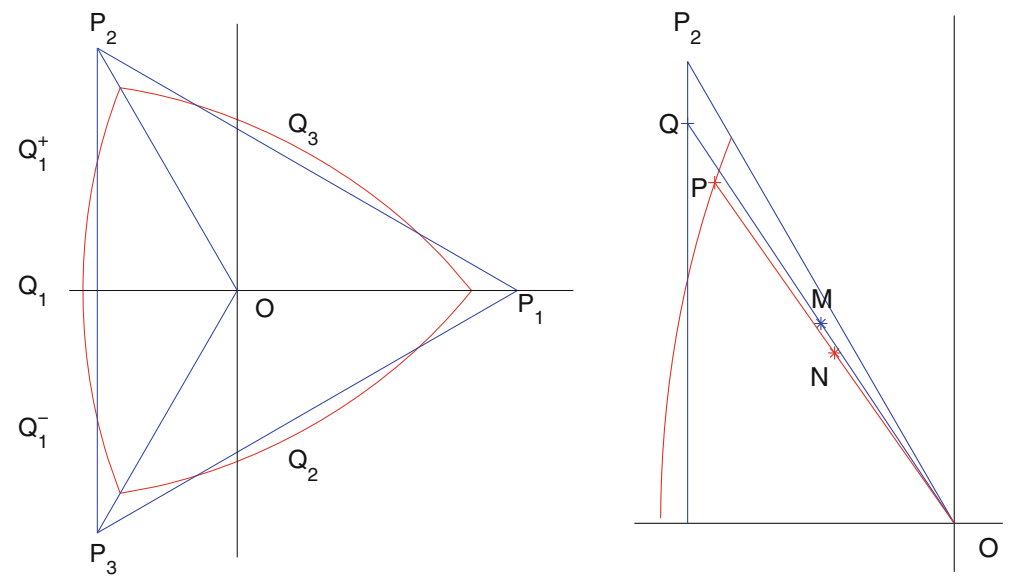

Figure 2. Left the equilateral triangle $T=P_{1} P_{2} P_{3}$, the curved triangle $\mathcal{T}$ and the zones $\mathcal{Q}_{1}, \mathcal{Q}_{1}^{+}, \mathcal{Q}_{1}^{-}, \mathcal{Q}_{2}, \mathcal{Q}_{3} ;$ Right the action of the transform $U$ : points $N$ and $P$ are the images of $M$ and $Q$, respectively

$$
\mathcal{Q}_{3}=\left\{(x, y) \in \mathbb{R}^{2}, \quad y>0, y \geq-\sqrt{3} x\right\}
$$

that are bounded by these line segments.

We focus for the moment on the region $\mathcal{Q}_{1}^{+}$defined by

$$
\mathcal{Q}_{1}^{+}=\left\{(x, y) \in \mathbb{R}^{2}, x<0,0 \leq y \leq-\sqrt{3} x\right\} \subset \mathcal{Q}_{1}
$$

and take a point $M=\left(x_{M}, y_{M}\right)=\left(x_{M}, m x_{M}\right) \in \mathcal{Q}_{1}^{+}$, where $m \in[-\sqrt{3}, 0]$ is a parameter, see Fig. 2, right. We want to define the map $U$ in such a way that each half-line $d_{m}$ of equation $y=m x(m \in[-\sqrt{3}, 0])$ is mapped onto the half-line $d_{\varphi(m)}$ of equation $y=\varphi(m) x$, where $\varphi:[-\sqrt{3}, 0] \rightarrow \mathbb{R}$ is a $C^{1}$ function that satisfies

$$
\varphi(0)=0, \varphi(-\sqrt{3})=-\sqrt{3}, \text { and }-\sqrt{3} \leq \varphi(m) \leq 0 \text { for } m \in[-\sqrt{3}, 0] .
$$

We denote by $\left(x_{N}, y_{N}\right)$ the coordinates of the point $N=U(M)$. Let $Q$ be the intersection of $O M$ with the triangle $T$ (see Fig. 2, right). The point $Q$ has the coordinates $\left(x_{Q}, y_{Q}\right)=(-\alpha,-m \alpha)$, where $\alpha$ is given in $(2.5)$, and the line $O N$ has the equation $y=\varphi(m) x$, i.e., $N=\left(x_{N}, \varphi(m) x_{N}\right)$. Further, let the point $P=\left(x_{P}, y_{P}\right)=\left(x_{P}, \varphi(m) x_{P}\right)$ be the intersection of $O N$ with the curved triangle $\mathcal{T}$. Thus, the coordinates of $P$ satisfy the equations (2.4). Replacing $x$ by the parameter $t=t_{P} \in[0,1]$ in $(2.4)$, we obtain

$$
x_{P}=\frac{\left(-t_{P}-\sqrt{1-t_{P}^{2}}\right)}{\sqrt{3+\sqrt{3}\left(t_{P}+\sqrt{1-t_{P}^{2}}\right)}},
$$




$$
y_{P}=\frac{\sqrt{3}\left(-t_{P}+\sqrt{1-t_{P}^{2}}\right)}{\left.\sqrt{3+\sqrt{3}\left(t_{P}+\sqrt{1-t_{P}^{2}}\right.}\right)},
$$

and from $y_{P}=\varphi(m) x_{P}$ we have

$$
\varphi(m)=\frac{y_{P}}{x_{P}}=\frac{\sqrt{3}\left(t_{P}-\sqrt{1-t_{P}^{2}}\right)}{t_{P}+\sqrt{1-t_{P}^{2}}} .
$$

From this equality we can determine $t_{P}$ as

$$
t_{P}=\frac{\varphi(m)+\sqrt{3}}{\sqrt{2} \sqrt{\varphi^{2}(m)+3}},
$$

and further, if we replace $t_{P}$ in (3.1) and (3.2), we obtain the coordinates of $P$ in the form

$$
\begin{aligned}
& x_{P}=-\frac{\sqrt{2}}{\sqrt{3+\varphi^{2}(m)+\sqrt{2\left(3+\varphi^{2}(m)\right)}}}, \\
& y_{P}=-\frac{\sqrt{2} \varphi(m)}{\sqrt{3+\varphi^{2}(m)+\sqrt{2\left(3+\varphi^{2}(m)\right)}}} .
\end{aligned}
$$

Some simple calculations yield the Euclidean distances

$$
\begin{aligned}
& O M=x_{M} \sqrt{1+m^{2}}, \\
& O Q=\alpha \sqrt{1+m^{2}}, \\
& O N=x_{N} \sqrt{1+\varphi^{2}(m)}, \\
& O P=\sqrt{2}\left(1-\frac{\sqrt{2}}{\sqrt{3+\varphi^{2}(m)}}\right)^{1 / 2},
\end{aligned}
$$

where $\alpha$ is given in (2.5). In order to simplify the determination of the map $U$, we suppose that

$$
\frac{O N}{O P}=\frac{O M}{O Q} .
$$

From the above calculations we then obtain $O N=\frac{x_{M}}{\alpha} O P$ and hence

$$
\begin{aligned}
& x_{N}=\frac{x_{M}}{\alpha} \frac{\sqrt{2}}{\sqrt{1+\varphi^{2}(m)}} \sqrt{1-\frac{\sqrt{2}}{\sqrt{3+\varphi^{2}(m)}}}, \\
& y_{N}=\varphi(m) x_{N}=\frac{x_{M}}{\alpha} \frac{\sqrt{2} \varphi(m)}{\sqrt{1+\varphi^{2}(m)}} \sqrt{1-\frac{\sqrt{2}}{\sqrt{3+\varphi^{2}(m)}}} .
\end{aligned}
$$


Thus, the map $U$ is now completely described by means of the function $\varphi$, and we obtain that $U$ maps the point $(x, y) \in \mathcal{Q}_{1}^{+}$onto the point $(X, Y)$ given by

$$
\begin{aligned}
& X=\frac{x}{\alpha} \frac{\sqrt{2}}{\sqrt{1+\varphi^{2}\left(\frac{y}{x}\right)}} \sqrt{1-\frac{\sqrt{2}}{\sqrt{3+\varphi^{2}\left(\frac{y}{x}\right)}}}, \\
& Y=\frac{x}{\alpha} \frac{\sqrt{2} \varphi\left(\frac{y}{x}\right)}{\sqrt{1+\varphi^{2}\left(\frac{y}{x}\right)}} \sqrt{1-\frac{\sqrt{2}}{\sqrt{3+\varphi^{2}\left(\frac{y}{x}\right)}}} .
\end{aligned}
$$

Next we have to ensure the area preserving property of $U$ by a suitable determination of $\varphi$. For this purpose, we define the function $\varphi$ such that ${ }^{1}$ the Jacobian of $U$ is 1 . After simplification, the Jacobian writes as

$$
J(U)=\operatorname{det}\left(\begin{array}{ll}
\frac{\partial X}{\partial x} & \frac{\partial X}{\partial y} \\
\frac{\partial Y}{\partial x} & \frac{\partial Y}{\partial y}
\end{array}\right)=\frac{2}{\alpha^{2}} \frac{\varphi^{\prime}\left(\frac{y}{x}\right)}{3+\varphi^{2}\left(\frac{y}{x}\right)+\sqrt{2} \sqrt{3+\varphi^{2}\left(\frac{y}{x}\right)}} .
$$

For solving the equation $J(U)=1$ we again substitute $m:=\frac{y}{x}$, and thus, in the considered case $0 \leq y \leq-\sqrt{3} x$ we have $m \in[-\sqrt{3}, 0]$. Hence, with the simplified notation $\varphi=\varphi(m)$, we get

$$
\frac{\varphi^{\prime}}{3+\varphi^{2}+\sqrt{2} \sqrt{3+\varphi^{2}}}=\frac{\alpha^{2}}{2} \text {. }
$$

Integration gives

$$
\arctan \varphi-\arctan \frac{\sqrt{2} \varphi}{\sqrt{3+\varphi^{2}}}=\frac{\alpha^{2}}{2} m+C .
$$

The condition $\varphi(0)=0$ yields $C=0$. Next, in order to determine $\varphi$ we use the formula

$$
\arctan a-\arctan b=\arctan \frac{a-b}{1+a b} \quad \forall a, b \in \mathbb{R}, a b>-1,
$$

and we further obtain

$$
\frac{\varphi\left(\sqrt{3+\varphi^{2}}-\sqrt{2}\right)}{\sqrt{3+\varphi^{2}}+\sqrt{2} \varphi^{2}}=\tan \frac{\alpha^{2} m}{2} .
$$

To simplify this term, we introduce the notation

$$
\eta=\tan \frac{\alpha^{2} m}{2}=\tan \frac{\pi m}{12 \sqrt{3}}
$$

and equality (3.6) yields

$$
\sqrt{3+\varphi^{2}}(\varphi-\eta)=\sqrt{2} \varphi(\eta \varphi+1)
$$

We must have $\varphi>\eta$ and with this condition, (3.7) is equivalent with

$$
\left(3+\varphi^{2}\right)(\varphi-\eta)^{2}=2 \varphi^{2}(\eta \varphi+1)^{2}
$$

\footnotetext{
${ }^{1}$ We recall that the area of a transformed region $D$ is $\mathcal{A}(U(D))=|J(U)| \mathcal{A}(D)$.
} 


$$
\left(\varphi^{2}+1\right)\left(\left(1-2 \eta^{2}\right) \varphi^{2}-6 \eta \varphi+3 \eta^{2}\right)=0
$$

which gives

$$
\varphi_{1,2}=\varphi_{1,2}(m)=\frac{3 \eta \pm \sqrt{6} \eta \sqrt{1+\eta^{2}}}{1-2 \eta^{2}} .
$$

To simplify the formulas, we denote $\delta=\frac{\alpha^{2} m}{2}=\frac{\pi m}{12 \sqrt{3}}=\frac{\pi y}{12 \sqrt{3} x}$ and with $\eta\left(1+\eta^{2}\right)^{-1 / 2}=\sin \delta$ and $\left(1+\eta^{2}\right)^{-1 / 2}=\cos \delta$ we further calculate

$$
\varphi_{1,2}(m)=\varphi_{1,2}\left(\frac{y}{x}\right)=\frac{\sqrt{3} \eta}{\sqrt{3} \mp \sqrt{2} \sqrt{1+\eta^{2}}}=\frac{\sqrt{3} \sin \delta}{\sqrt{3} \cos \delta \pm \sqrt{2}} .
$$

If we take into account the condition $\varphi(-\sqrt{3})=-\sqrt{3}$ and the equality

$$
\sin \frac{\pi}{12}=\sqrt{3} \cos \frac{\pi}{12}-\sqrt{2},
$$

the only convenient solution is

$$
\varphi\left(\frac{y}{x}\right)=\frac{\sqrt{3} \sin \delta}{\sqrt{3} \cos \delta-\sqrt{2}}, \quad \text { with } \delta=\frac{\pi y}{12 \sqrt{3} x} .
$$

Finally, in order to give the explicit presentation of the area preserving map $U$, we need to replace $\varphi$ in the equations (3.3), (3.4). Some straightforward calculations show that

$$
\begin{aligned}
\sqrt{1+\varphi^{2}} & =\frac{\sqrt{5-2 \sqrt{6} \cos \delta}}{\sqrt{3} \cos \delta-\sqrt{2}}, \\
\sqrt{3+\varphi^{2}} & =\frac{\sqrt{3}(\sqrt{3}-\sqrt{2} \cos \delta)}{\sqrt{3} \cos \delta-\sqrt{2}}, \\
1-\frac{\sqrt{2}}{\sqrt{3+\varphi^{2}}} & =\frac{5-2 \sqrt{6} \cos \delta}{3-\sqrt{6} \cos \delta} .
\end{aligned}
$$

Hence, for $(x, y) \in \mathcal{Q}_{1}^{+}$, the formulas for the desired area preserving map $U(x, y)=(X, Y)$ are given by

$$
\begin{aligned}
& X=\frac{2 \sqrt{3} x}{\sqrt{\pi}} \frac{\sqrt{3} \cos \delta-\sqrt{2}}{\sqrt{\sqrt{3}-\sqrt{2} \cos \delta}}=: f(x, y), \\
& Y=\frac{6 x}{\sqrt{\pi}} \frac{\sin \delta}{\sqrt{\sqrt{3}-\sqrt{2} \cos \delta}}=: g(x, y), \text { with } \delta=\frac{y \pi}{12 \sqrt{3} x} .
\end{aligned}
$$

Similar arguments for the region $\mathcal{Q}_{1}^{-}=\left\{(x, y) \in \mathbb{R}^{2}, x<0, \sqrt{3} x \leq y \leq 0\right\}$ show that these formulas also hold for $(x, y) \in \mathcal{Q}_{1}^{-}$, so we can conclude that the image $U(x, y)=(X, Y)$ of a point $(x, y)$ in the region

$$
\mathcal{Q}_{1}=\mathcal{Q}_{1}^{+} \cup \mathcal{Q}_{1}^{-}=\left\{(x, y) \in \mathbb{R}^{2}, \quad x<0, \sqrt{3} x \leq y \leq-\sqrt{3} x\right\}
$$

is given by formulas (3.8), (3.9). 
For the regions $\mathcal{Q}_{2}$ and $\mathcal{Q}_{3}$ we may use the formulas (3.8), (3.9) after performing some rotations. More precisely, we need the rotation matrix

$$
\mathcal{A}=\mathcal{A}_{\frac{2 \pi}{3}}=\left(\begin{array}{cc}
\cos \frac{2 \pi}{3} & -\sin \frac{2 \pi}{3} \\
\sin \frac{2 \pi}{3} & \cos \frac{2 \pi}{3}
\end{array}\right)=\left(\begin{array}{cc}
-\frac{1}{2} & -\frac{\sqrt{3}}{2} \\
\frac{\sqrt{3}}{2} & -\frac{1}{2}
\end{array}\right) .
$$

We denote by $U_{1}, U_{2}, U_{3}$ the restrictions of $U$ to $\mathcal{Q}_{1}, \mathcal{Q}_{2}, \mathcal{Q}_{3}$, respectively. Then, for $(x, y) \in \mathcal{Q}_{2}$ we have

$$
\left(\begin{array}{l}
x^{\prime} \\
y^{\prime}
\end{array}\right)=\mathcal{A}^{-1} \cdot\left(\begin{array}{l}
x \\
y
\end{array}\right) \in \mathcal{Q}_{1},
$$

therefore, to $\left(x^{\prime}, y^{\prime}\right)$ we can apply $U$ defined by the formulas (3.8), (3.9) and obtain' $\left(X^{\prime}, Y^{\prime}\right) \in \mathcal{Q}_{1}$,

$$
X^{\prime}=f\left(x^{\prime}, y^{\prime}\right), \quad Y^{\prime}=g\left(x^{\prime}, y^{\prime}\right), \text { or }\left(\begin{array}{l}
X^{\prime} \\
Y^{\prime}
\end{array}\right)=U_{1}\left(x^{\prime}, y^{\prime}\right) .
$$

Finally, if we apply to $\left(X^{\prime}, Y^{\prime}\right)$ a rotation of $\frac{2 \pi}{3}$ we obtain $\left(X^{\prime \prime}, Y^{\prime \prime}\right) \in \mathcal{Q}_{2}$ defined by

$$
\left(\begin{array}{l}
X^{\prime \prime} \\
Y^{\prime \prime}
\end{array}\right):=U(x, y)=U_{2}(x, y)=\mathcal{A} \cdot U_{1}\left(x^{\prime}, y^{\prime}\right)=\mathcal{A} \cdot U_{1}\left(\mathcal{A}^{-1} \cdot\left(\begin{array}{l}
x \\
y
\end{array}\right)\right) .
$$

Similarly, for $(x, y) \in \mathcal{Q}_{3}$ we obtain

$$
\left(\begin{array}{l}
X^{\prime \prime} \\
Y^{\prime \prime}
\end{array}\right):=U(x, y)=U_{3}(x, y)=\mathcal{A}^{-1} \cdot U_{1}\left(x^{\prime}, y^{\prime}\right)=\mathcal{A}^{-1} U_{1}\left(\mathcal{A} \cdot\left(\begin{array}{l}
x \\
y
\end{array}\right)\right) .
$$

In conclusion, the area preserving map $U$ which maps equilateral triangles onto curved triangles can be written as follows:

- $\operatorname{For}(x, y) \in \mathcal{Q}_{1}=\left\{(x, y) \in \mathbb{R}^{2}, \sqrt{3} x \leq y \leq-\sqrt{3} x\right\}$,

$$
\begin{aligned}
& (x, y) \longmapsto U_{1}(x, y)=(X, Y):= \\
& \quad\left(\frac{2 \sqrt{3} x}{\sqrt{\pi}} \frac{\sqrt{3} \cos \delta-\sqrt{2}}{\sqrt{\sqrt{3}-\sqrt{2} \cos \delta}}, \frac{6 x}{\sqrt{\pi}} \frac{\sin \delta}{\sqrt{\sqrt{3}-\sqrt{2} \cos \delta}}\right) ;
\end{aligned}
$$

- $\operatorname{For}(x, y) \in \mathcal{Q}_{2}=\left\{(x, y) \in \mathbb{R}^{2}, y<0, y \leq \sqrt{3} x\right\}$,

$$
(x, y) \longmapsto(X, Y)^{T}=\mathcal{A} \cdot U_{1}\left(\mathcal{A}^{-1} \cdot\left(\begin{array}{l}
x \\
y
\end{array}\right)\right) ;
$$

- $\operatorname{For}(x, y) \in \mathcal{Q}_{3}=\left\{(x, y) \in \mathbb{R}^{2}, y>0, y \geq-\sqrt{3} x\right\}$,

$$
(x, y) \longmapsto(X, Y)^{T}=\mathcal{A}^{-1} \cdot U_{1}\left(\mathcal{A} \cdot\left(\begin{array}{l}
x \\
y
\end{array}\right)\right)
$$

with $\delta=\frac{y \pi}{12 \sqrt{3} x}$ and $\mathcal{A}=\left(\begin{array}{cc}-\frac{1}{2} & -\frac{\sqrt{3}}{2} \\ \frac{\sqrt{3}}{2} & -\frac{1}{2}\end{array}\right)$. Figure 3 shows two examples of planar grids. 

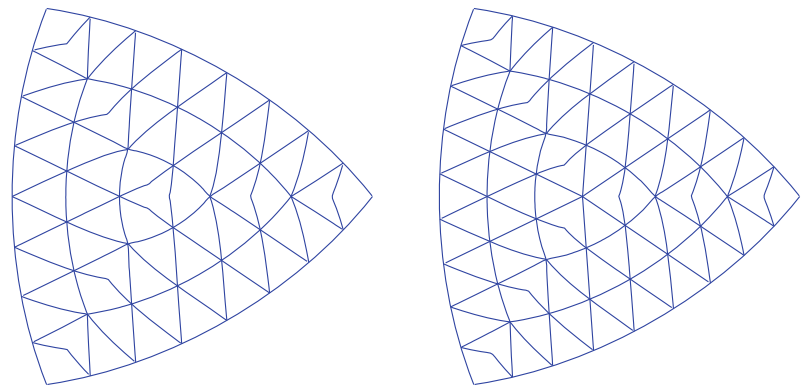

FiguRE 3. Two examples of planar grids of $\mathcal{T}$, images of grids of $T$ constructed with 7 and 8 equidistant parallel lines

\section{Mapping $\boldsymbol{F}_{i}$ onto $\widetilde{\boldsymbol{F}}_{\boldsymbol{i}}$}

The area preserving map $U: \mathbb{R}^{2} \rightarrow \mathbb{R}^{2}$ derived in Sect. 3 depends on two variables. We want to use it for mapping $F_{i}$ onto $\widetilde{F}_{i}$, where both $F_{i}$ and $\widetilde{F}_{i}$ are included in the same plane of $\mathbb{R}^{3}$.

We denote by $\mathcal{P}_{-1}$ the plane $z=-1$ and define $\widetilde{U}: \mathcal{P}_{-1} \rightarrow \mathcal{P}_{-1}$ by

$$
\widetilde{U}(x, y,-1)=(X, Y,-1) \text {, with }(X, Y)=U(x, y) \text {, for all }(x, y) \in \mathbb{R}^{2} \text {. }
$$

This map allows us to write the formulas for the functions $\mathcal{U}_{i}: F_{i} \rightarrow \widetilde{F}_{i}$, for $i=1, \ldots, 8$, which map triangles (i.e., the faces of the octahedron) onto curved triangles.

As shown in Sect. 2, we observe that

$$
\mathcal{R} \cdot(|x|,|y|,|z|)^{T} \in \mathcal{P}_{-1},
$$

in each of the cases $(x, y, z) \in F_{i}, i=1, \ldots, 8$, where $\mathcal{R}$ is given in (2.2). Therefore, $\widetilde{U}\left(\mathcal{R} \cdot(|x|,|y|,|z|)^{T}\right) \in \mathcal{P}_{-1}$ and

$$
\mathcal{R}^{-1} \cdot \widetilde{U}\left(\mathcal{R} \cdot(|x|,|y|,|z|)^{T}\right) \in \widetilde{F}_{1} \text {, for }(x, y, z) \in F_{1} .
$$

So, we define $\mathcal{U}_{1}: F_{1} \rightarrow \widetilde{F}_{1}$,

$$
\mathcal{U}_{1}(x, y, z)=\mathcal{R}^{-1} \cdot \widetilde{U}\left(\mathcal{R} \cdot(|x|,|y|,|z|)^{T}\right)=:(X, Y, Z), \text { for }(x, y, z) \in F_{1},(4.1)
$$

and further, with the notations in (4.1), the required applications $\mathcal{U}_{i}: F_{i} \rightarrow \widetilde{F}_{i}$ are defined as follows:

$$
\begin{aligned}
& \mathcal{U}_{2}(x, y, z)=(X, Y,-Z), \quad \mathcal{U}_{3}(x, y, z)=(X,-Y, Z), \\
& \mathcal{U}_{4}(x, y, z)=(X,-Y,-Z), \quad \mathcal{U}_{5}(x, y, z)=(-X, Y, Z), \\
& \mathcal{U}_{6}(x, y, z)=(-X, Y,-Z), \quad \mathcal{U}_{7}(x, y, z)=(-X,-Y, Z), \\
& \mathcal{U}_{8}(x, y, z)=(-X,-Y,-Z)
\end{aligned}
$$

Finally, we denote by $\mathcal{U}: \mathbb{K} \rightarrow \cup_{i=1}^{8} \widetilde{F}_{i}$ the map satisfying the condition $\mathcal{U}=\mathcal{U}_{i}$ on $F_{i}$. 


\section{Mapping the Curved Triangles onto the Sphere}

The complete mapping from the regular octahedron $\mathbb{K}$ to the sphere $\mathbb{S}^{2}$ is now described in two steps. In the first step, each face $F_{i}$ of $\mathbb{K}$ will be mapped onto a planar domain $\widetilde{F}_{i}$, bounded by a curved triangle $\mathcal{T}_{i}$, using the transformation $\mathcal{U}$ constructed in the previous section. In the second step, each $\widetilde{F}_{i}$ will be mapped onto $\mathcal{F}_{i} \subseteq \mathbb{S}^{2}$ by the inverse Lambert azimuthal projection, with respect to $G_{i}=U\left(W_{i}\right)$, where $W_{i}$ is the center of mass of $F_{i}$. Obviously

$$
\bigcap_{i=1}^{8} \operatorname{int} \mathcal{F}_{i}=\emptyset \text { and } \bigcup_{i=1}^{8} \mathcal{F}_{i}=\mathbb{S}^{2} \text {. }
$$

Applying the inverse Lambert projection $L_{S}^{-1}$, the point $(X, Y,-1)$, situated in the tangent plane to $\mathbb{S}^{2}$ at the South Pole $S$, maps onto $\left(x_{L}, y_{L}, z_{L}\right) \in \mathbb{S}^{2}$ given by

$$
\begin{aligned}
& x_{L}=\sqrt{1-\frac{X^{2}+Y^{2}}{4}} X, \\
& y_{L}=\sqrt{1-\frac{X^{2}+Y^{2}}{4}} Y, \\
& z_{L}=\frac{X^{2}+Y^{2}}{2}-1 .
\end{aligned}
$$

Thus, the application $L_{G_{i}}^{-1} \circ \mathcal{U}$ maps the face $F_{i}$ of the octahedron onto $\mathcal{F}_{i} \subset \mathbb{S}^{2}$, for $i=1, \ldots, 8$. From $(2.3)$ we have $L_{G_{1}}^{-1}=\mathcal{R}^{-1} \circ L_{S}^{-1} \circ \mathcal{R}$, and for obtaining the other projections $L_{G_{i}}^{-1}$ we proceed as in the previous section. Again, $\mathcal{R} \cdot(|x|,|y|,|z|)^{T} \in \mathcal{P}_{-1}$, therefore we use formulas (5.1)-(5.3) and we have

$$
\left(x_{L}, y_{L}, z_{L}\right)=L_{S}^{-1}\left(\mathcal{R} \cdot(|x|,|y|,|z|)^{T}\right) .
$$

Further,

$$
(X, Y, Z)^{T}:=\mathcal{R}^{-1} \cdot\left(x_{L}, y_{L}, z_{L}\right)^{T} \in \mathcal{F}_{1},
$$

and then, with these notations, for the other cases we obtain the following expressions for $L_{G_{i}}^{-1}: \widetilde{F}_{i} \rightarrow \mathcal{F}_{i}$ :

$$
\begin{aligned}
& L_{G_{2}}^{-1}(x, y, z)=(X, Y,-Z), \quad L_{G_{3}}^{-1}(x, y)=(X,-Y, Z), \\
& L_{G_{4}}^{-1}(x, y, z)=(X,-Y,-Z), \quad L_{G_{5}}^{-1}(x, y)=(-X, Y, Z), \\
& L_{G_{6}}^{-1}(x, y, z)=(-X, Y,-Z), \quad L_{G_{7}}^{-1}(x, y)=(-X,-Y, Z), \\
& L_{G_{8}}^{-1}(x, y, z)=(-X,-Y,-Z) .
\end{aligned}
$$

Figure 4 shows some grids of the sphere, where a regular partition of the faces of the octahedron into 4, 16, 25 and 64 equal area triangles has been applied. 

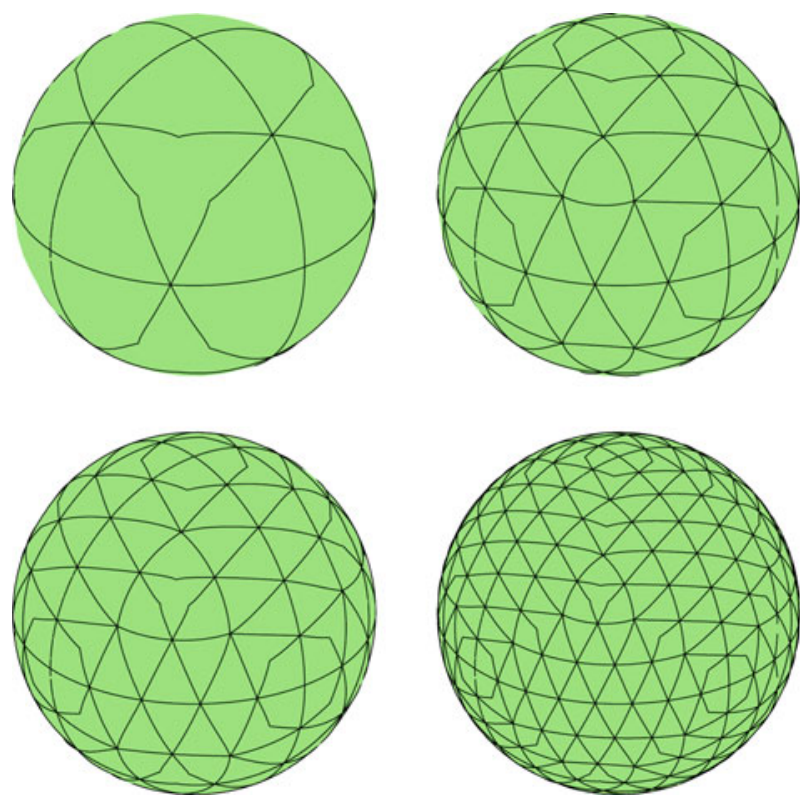

Figure 4. Grids on the sphere

\section{The Inverse Map}

To make the area preserving map $\mathcal{U}: \mathbb{K} \rightarrow \cup_{i=1}^{8} \widetilde{F}_{i}$ applicable in practice, we also need to derive a closed simple form for the inverse mapping $\mathcal{U}^{-1}$. In view of the above considerations we can restrict to the calculation of the inverse $U^{-1}$ of the area-preserving map proposed in Sect. 3, since the mapping of the portions $\mathcal{F}_{i}$ to $\widetilde{F}_{i}$ is obtained by the azimuthal Lambert projection, and the area preserving mapping of the curved triangle $\widetilde{F}_{i}$ to the faces $F_{i}$ of the octahedron is completely understood, if we find $U^{-1}: T \rightarrow \mathcal{T}$ with the twodimensional triangles considered in Sect. 3, see Fig. 2. Further, we can restrict the calculations again to the region $\mathcal{Q}_{1}$, and the complete map $U^{-1}$ is then derived by rotation.

With the notation

$$
\delta=\frac{y \pi}{12 \sqrt{3} x}
$$

(excluding the case $x=0$ ), from (3.8) and (3.9) we have

$$
X^{2}=\frac{12 x^{2}}{\pi} \frac{\left(3 \cos ^{2} \delta+2-2 \sqrt{6} \cos \delta\right)}{(\sqrt{3}-\sqrt{2} \cos \delta)}, \quad Y^{2}=\frac{36 x^{2}}{\pi} \frac{\left(\sin ^{2} \delta\right)}{(\sqrt{3}-\sqrt{2} \cos \delta)} .
$$


In particular, we obtain

$$
\begin{aligned}
X^{2}+Y^{2} & =\frac{12 x^{2}}{\pi} \frac{(5-2 \sqrt{6} \cos \delta)}{(\sqrt{3}-\sqrt{2} \cos \delta)}, \\
X^{2}+\frac{1}{3} Y^{2} & =\frac{12 x^{2}}{\pi}(\sqrt{3}-\sqrt{2} \cos \delta) .
\end{aligned}
$$

We define

$$
B:=\frac{X^{2}+\frac{1}{3} Y^{2}}{X^{2}+Y^{2}}=\frac{\left(3+2 \cos ^{2} \delta-2 \sqrt{6} \cos \delta\right)}{(5-2 \sqrt{6} \cos \delta)}
$$

and derive the relation

$$
\cos ^{2} \delta+\sqrt{6}(B-1) \cos \delta+\frac{(3-5 B)}{2}=0
$$

with the solutions

$$
(\cos \delta)_{1,2}=\frac{-\sqrt{3}(B-1) \pm \sqrt{B(3 B-1)}}{\sqrt{2}} .
$$

Since $B \in\left(\frac{1}{3}, 1\right)$, the only convenient solution is $\cos \delta=\frac{\sqrt{3}(1-B)+\sqrt{B(3 B-1)}}{\sqrt{2}}$. Then, from

$$
1-B=\frac{2 Y^{2}}{3\left(X^{2}+Y^{2}\right)} \text { and } 3 B-1=\frac{2 X^{2}}{X^{2}+Y^{2}}
$$

it follows that

$$
\cos \delta=\frac{\sqrt{2} Y^{2}+X \sqrt{3 X^{2}+Y^{2}}}{\sqrt{3}\left(X^{2}+Y^{2}\right)} .
$$

For simplicity, we introduce the notation $w:=Y / X$. Since $(X, Y) \in \mathcal{Q}_{1}$, we have $w \in[-\sqrt{3}, \sqrt{3}]$ and

$$
\delta=\arccos \left(\frac{\sqrt{2} w^{2}+\sqrt{3+w^{2}}}{\sqrt{3}\left(1+w^{2}\right)}\right)=\arccos \left(\frac{\sqrt{2} w^{2}}{\sqrt{3}\left(w^{2}+1\right)}+\frac{\sqrt{w^{2}+3}}{\sqrt{3}\left(w^{2}+1\right)}\right) .
$$

Now we use the identity

$$
\arccos \left(a b+\sqrt{1-a^{2}} \sqrt{1-b^{2}}\right)=\left\{\begin{array}{l}
\arccos a-\arccos b \text { for } a<b \\
\arccos b-\arccos a \text { for } a \geq b
\end{array}\right.
$$

where $a=\frac{\sqrt{2} w}{\sqrt{3\left(1+w^{2}\right)}}$ and $b=\frac{w}{\sqrt{1+w^{2}}}$, and we obtain

$$
\delta=\frac{\pi}{12 \sqrt{3}} \frac{y}{x}=\left\{\begin{array}{l}
\arccos \left(\frac{\sqrt{2} w}{\sqrt{3\left(1+w^{2}\right)}}\right)-\arccos \left(\frac{w}{\sqrt{1+w^{2}}}\right) \text { for } w \in(0, \sqrt{3}], \\
-\arccos \left(\frac{\sqrt{2} w}{\sqrt{3\left(1+w^{2}\right)}}\right)+\arccos \left(\frac{w}{\sqrt{1+w^{2}}}\right) \text { for } w \in[-\sqrt{3}, 0] .
\end{array}\right.
$$


For the calculation of $x$ from $X$ and $Y$, we use the equalities in (6.1) and (6.2) yielding

$$
\begin{aligned}
x^{2} & =\frac{\pi}{12} \frac{\left(X^{2}+\frac{1}{3} Y^{2}\right)}{(\sqrt{3}-\sqrt{2} \cos \delta)} \\
& =\frac{\pi}{12 \sqrt{3}} \frac{\left(3 X^{2}+Y^{2}\right)\left(X^{2}+Y^{2}\right)}{3\left(X^{2}+Y^{2}\right)-\sqrt{2}\left(\sqrt{2} Y^{2}+X \sqrt{3 X^{2}+Y^{2}}\right)} \\
& =\frac{\pi}{12 \sqrt{3}} \frac{\sqrt{3 X^{2}+Y^{2}}\left(X^{2}+Y^{2}\right)}{\sqrt{3 X^{2}+Y^{2}}-\sqrt{2} X} \\
& =\frac{\pi}{12 \sqrt{3}} \sqrt{3 X^{2}+Y^{2}}\left(\sqrt{3 X^{2}+Y^{2}}+\sqrt{2} X\right) .
\end{aligned}
$$

Finally, from $(6.3)$ we find $y=|y|(\operatorname{sign} Y)$ with

$$
\begin{aligned}
|y| & =\frac{12 \sqrt{3}}{\pi}|x||\delta|=\frac{12 \sqrt{3}}{\pi}|x|\left|\arccos \left(\frac{\sqrt{2} w^{2}}{\sqrt{3}\left(w^{2}+1\right)}+\frac{\sqrt{w^{2}+3}}{\sqrt{3}\left(w^{2}+1\right)}\right)\right| \\
& =\frac{12 \sqrt{3}}{\pi}|x|\left|\arccos \frac{\sqrt{2} Y}{\sqrt{3\left(X^{2}+Y^{2}\right)}}-\arccos \frac{Y}{\sqrt{X^{2}+Y^{2}}}\right| .
\end{aligned}
$$

The obtained formulas for $x$ and $y$ form an explicit representation of the inverse map $U^{-1}$ in the region $\mathcal{Q}_{1}$. For the other two regions, the map is directly obtained using the rotation matrix $\mathcal{A}$ in Sect. 3 .

\section{Acknowledgements}

The work has been funded by a Grant for bilateral cooperation (PL 170/14-1) of the German Research Foundation (DFG). This is gratefully acknowledged.

Open Access. This article is distributed under the terms of the Creative Commons Attribution License which permits any use, distribution, and reproduction in any medium, provided the original author(s) and the source are credited.

\section{References}

[1] Alexander, R.: On the sume of distances between $N$ points on the sphere. Acta Mathematica. 23, 443-448 (1972)

[2] Grafared, E.W., Krumm, F.W.: Map Projections, Cartographic Information Systems. Springer, Berlin (2006)

[3] Górski, K.M., Wandelt, B.D., Hivon, E., Banday, A.J., Hansen, F.K., Reinecke, M., Bartelmann, M.: HEALPix: A framework for high-resolution discretization and fast analysis of data distributed on the sphere. Astrophys. J. 622(2), 759 (2005)

[4] Leopardi, P.: A partition of the unit sphere into regions of equal area and small diameter. Electron. Trans. Numer. Anal. 25, 309-327 (2006) 
[5] Roşca, D.: New uniform grids on the sphere. Astron. Astrophys. 520, A63 (2010)

[6] Roşca, D.: Uniform and refinable grids on elliptic domains and on some surfaces of revolution. Appl. Math. Comput. 217(19 ), 7812-7817 (2011)

[7] Roşca, D., Plonka, G.: Uniform spherical grids via equal area projection from the cube to the sphere. J. Comput. Appl. Math. 236, 1033-1041 (2011)

[8] Song, L., Kimerling, A.J., Sahr K.: Developing an equal area global grid by small circle subdivision. In: Goodchild, M., Kimerling, A.J. (eds.) Discrete Global Grids. National Center for Geographic Information \& Analysis, Santa Barbara (2002)

[9] Snyder, J.P.: Flattening the Earth. University of Chicago Press, Chicago (1990)

[10] Teanby, N.A.: An icosahedron-based method for even binning of globally distributed remote sensing data. Comput. Geosci. 32(9), 1442-1450 (2006)

[11] Tegmark, M.: An icosahedron-based method for pixelizing the celestial sphere. Astrophys. J. 470, L81-L84 (1996)

[12] Zhou,Y.M.: Arrangements of points on the sphere. PhD thesis, Mathematics, Tampa, FL (1995)

Daniela Roşca

Department of Mathematics

Technical University of Cluj-Napoca

str. Memorandumului 28

RO-400114 Cluj-Napoca

Romania

e-mail: Daniela.Rosca@math.utcluj.ro

Gerlind Plonka

Institute for Numerical and Applied Mathematics

University of Göttingen

Lotzestr. 16-18

37083 Göttingen

Germany

e-mail: plonka@math.uni-goettingen.de

Received: January 16, 2012.

Accepted: July 30, 2012. 